\title{
GELFAND-KIRILLOV DIMENSION IN JORDAN ALGEBRAS
}

\author{
C. MARTINEZ
}

\begin{abstract}
In this paper we study Gelfand-Kirillov dimension in Jordan algebras. In particular we will relate Gelfand-Kirillov (GK for short) dimensions of a special Jordan algebra and its associative enveloping algebra and also the GK dimension of a Jordan algebra and the GK dimension of its universal multiplicative enveloping algebra.
\end{abstract}

\section{INTRODUCTION}

Definition. Let $A$ be a finitely generated (not necessarily associative) algebra. Let $V$ be a finite dimensional $K$-vector space generating $A$ and let $V^{n}$ denote the linear span of all products of length $\leq n$ in elements of $V$. The Gelfand-Kirillov dimension of $A(G K \operatorname{dim}(A)$ for short) is defined by:

$$
\operatorname{GKdim}(A)=\limsup _{n \rightarrow \infty} \frac{\ln \left[\operatorname{dim} V^{n}\right]}{\ln n} .
$$

It is known that the above definition does not depend on the choice of a particular finite dimensional vector space generating $A$ (see [1] and [3]).

Clearly $G K \operatorname{dim}(A)=0$ if and only if $\operatorname{dim}_{K} A<\infty$ and, in fact, there are no algebras with $0<G K \operatorname{dim}(A)<1$ (see [3]). In the associative case Bergman proved that there are no associative algebras with $1<G K \operatorname{dim}(A)<2$ (this is not known in the non-associative case, not even for Jordan algebras). However there are algebras having dimension $s$ for every $2 \leq s$.

We will start relating GK dimensions of a special Jordan algebra $J$ and GKdim of its associative enveloping algebra $A$.

If $A$ is an associative algebra, $A^{(+)}$denotes the Jordan algebra associated with $A$, that is, the algebra with the same underlying vector space of $A$ and with the new product : $a \cdot b=\frac{1}{2}(a b+b a)$ (here the associative product in $A$ is denoted by juxtaposition). A Jordan algebra $J$ is said to be special if it is a subalgebra of $A^{(+)}$ for some associative algebra $A$. The associative subalgebra of $A$ generated by $J$ is the associative enveloping of the special Jordan algebra $J$.

So in section 1 we prove the following theorem.

Theorem 1.2. Let $J$ be a finitely generated special Jordan algebra and let $A$ be its associative enveloping algebra. Then $G K \operatorname{dim}(J)=G K \operatorname{dim}(A)$.

Received by the editors July 1, 1994.

1991 Mathematics Subject Classification. Primary 17C50, 17C99.

Key words and phrases. Jordan algebra, Gelfand-Kirillov dimension.

Partially supported by DGICYT, Ps. 90-0129.

(C)1996 American Mathematical Society 
For an arbitrary Jordan algebra $J$ let $M(J)$ be its universal multiplicative enveloping algebra (see [2] or section 2 of this paper).

The following theorem is proved in section 2 .

Theorem 2.2. $G K \operatorname{dim}(M(J) \leq 2 G K \operatorname{dim}(J)$.

The Example 2.1 given in the same section shows that the inequality above can be strict. However, for special Jordan algebras we get more precise information.

Theorem 2.4. Let $J$ be a finitely generated special Jordan algebra. Then

$$
G K \operatorname{dim}(M(J))=2 G K \operatorname{dim}(J) .
$$

Finally, in section 3 we discuss GK-dimensions of Jordan algebras, Jordan pairs and the Tits-Kantor-Koecher Lie algebras.

\section{Special Jordan ALGEBras}

We will consider $J$ a finitely generated Jordan algebra and $A$ its associative enveloping algebra, so $A$ is also finitely generated. We will need the following result which is due to Skosirskii:

Theorem 1.1 (Skosirskii, see [7]). Let $J$ be a finitely generated special Jordan algebra and let $A$ be its associative enveloping algebra. If $a_{1}, \ldots, a_{m}$ is a set of generators of $J$, then every element in $A$ can be written as a linear combination of elements in the form: $b a_{i_{1}} \ldots a_{i_{t}}$, where $i_{1}<\ldots<i_{t}$ and $b$ is a Jordan product of elements $a_{1}, \ldots, a_{m}$ or $b=1$.

With the above result we can now prove the first mentioned theorem.

Theorem 1.2. Let $J$ be a finitely generated special Jordan algebra and let $A$ be its associative enveloping algebra. Then $G K \operatorname{dim}(J)=G K \operatorname{dim}(A)$.

Proof. Let $V$ be a finite dimensional vector space over $K$ that generates $A$. Let $a_{1}, \ldots, a_{m}$ be a basis of $V$. By $V^{\bullet}$ we denote the vector space spanned by all Jordan products of length $\leq n$ of generators $a_{1}, \ldots, a_{m}$. Let $V^{n}$ be the vector space spanned by all associative products of length $\leq n$ in $A$ of generators $a_{1}, \ldots, a_{m}$. So $V^{\bullet n} \subseteq V^{n}$ and clearly

$$
\operatorname{GKdim}(J)=\limsup _{n \rightarrow \infty} \frac{\ln \left[\operatorname{dim} V^{\bullet n}\right]}{\ln n} \leq \limsup _{n \rightarrow \infty} \frac{\ln \left[\operatorname{dim} V^{n}\right]}{\ln n}=\operatorname{GKdim}(A) .
$$

As usual the associative product in $A$ is denoted by juxtaposition.

By the Theorem of Skosirskii, an arbitrary product $\pi$ of length $n$ can be expressed as a linear combination of elements in the form: $b a_{i_{1}} \ldots a_{i_{t}}$ where $i_{1}<\ldots<$ $i_{t}$ and $b$ is a Jordan product of $a_{1}, \ldots, a_{m}$ or $b=1$. Moreover, from the the proof of Skosirskii's theorem it follows that each summand $b a_{i_{1}} \ldots a_{i_{t}}$ has the same composition as $\pi$ (see [7]).

There are $2^{m}$ words $a_{i_{1}} \ldots a_{i_{t}}, i_{1}<\ldots<i_{t}$ (including the empty word).

Hence $\operatorname{dim} V^{n} \leq 2^{m}\left(\operatorname{dim} V^{\bullet n}+1\right) \quad$ and

$$
\begin{aligned}
\operatorname{GKdim}(A) & =\limsup _{n \rightarrow \infty} \frac{\ln \left[\operatorname{dim} V^{n}\right]}{\ln n} \leq \limsup _{n \rightarrow \infty} \frac{\ln \left[2^{m}\left(\operatorname{dim} V^{\bullet n}+1\right)\right]}{\ln n} \\
& \leq \limsup _{n \rightarrow \infty} \frac{\ln 2^{m}}{\ln n}+\limsup _{n \rightarrow \infty} \frac{\ln \left[\operatorname{dim} V^{\bullet n}+1\right]}{\ln n}=\operatorname{GKdim}(J) .
\end{aligned}
$$

Consequently $G K \operatorname{dim}(A)=G K \operatorname{dim}(J)$. 
Corollary 1.1. Let $J$ be a Jordan algebra (not necessarily special) and let $S(J)$ be its universal associative enveloping algebra. Then $G K \operatorname{dim}(S(J)) \leq G K \operatorname{dim}(J)$.

Proof. Let $\varphi: J \longrightarrow S(J)$ be a universal specialization. Then $S(J)$ is the associative enveloping of the algebra $\varphi(J)$. So Theorem 2.1 assures that $G K \operatorname{dim}(\varphi(J))$ $=G K \operatorname{dim}(S(J))$. But $\varphi(J)$ is an homomorphic image of $J$, and consequently $G K \operatorname{dim}(J) \geq G K \operatorname{dim}(\varphi(J))=G K \operatorname{dim}(S(J))$.

Corollary 1.2. Let $A$ be a finitely generated alternative algebra, char $K \neq 2$. Then $G K \operatorname{dim}(A)=G K \operatorname{dim}(R(A))$, where $R(A)$ is the right multiplication algebra of $A$.

Proof. Let $A$ and $A^{(+)}$denote the alternative algebra and its associated Jordan algebra, where product in $A^{(+)}$is given by: $a \cdot b=\frac{1}{2}(a b+b a)$.

It is known (see [8]) that $A^{(+)}$is also finitely generated.

We can assume the existence of a unit element in $A$.

Let $A^{*}$ be the linear span of right multiplications by elements of $A$. It is known that $A^{*}$ is also a Jordan algebra $\left(R(a) \circ R(b)=\frac{1}{2}(R(a) R(b)+R(b) R(a))=R(a . b)\right)$, $A^{*}$ is isomorphic to $A^{(+)}$and $R(A)$ is the associative enveloping algebra of the special Jordan algebra $A^{*}$.

By Theorem 1.2, GKdim $\left(A^{(+)}\right)=G K \operatorname{dim}\left(A^{*}\right)=G K \operatorname{dim}(R(A))$.

But clearly $G K \operatorname{dim}\left(A^{(+)}\right) \leq G K \operatorname{dim}(A)$ ( since if $W$ is a finite dimensional vector space generating $A^{(+)}$, it also generates $A$ and $W^{\bullet n} \subseteq W^{n}$ for every natural number $n$, with the same notation as in Theorem 1.2).

Also, if $R(A)$ is generated by the finite dimensional $K$-vector space $U$, then $W^{n} \subseteq 1 . U^{n}$. So $G K \operatorname{dim}(A) \leq G K \operatorname{dim}(R(A))$ and the equality follows.

Remark 1.1. The above result is also true when $\operatorname{char} K=2$. The proof follows the same lines that the one given in Theorem 1.2, but instead of the Skosirskii result mentioned in Theorem 1.1., it would use the following result of [9] (Lemma 6, p. $275)$.

Lemma 1.1. Any element from the right multiplication algebra $R(A l t[X])$ of the algebra Alt $[X]$ is a linear combination of elements of the form $R\left(u_{1}\right) R\left(u_{2}\right) \ldots$ $R\left(u_{n}\right) R(x)$, where $x$ is an arbitrary element of the algebra Alt $[X]$ and $u_{1}, \ldots, u_{n}$ are mutually distinct regular $r_{1}$-words from the set $X$. In addition, the operators $R\left(u_{1}\right), \ldots, R\left(u_{n}\right)$ can be missing.

\section{The universal multiplicative ENVEloping algeBras}

Let $B=B(X)$ be a free bimodule over a Jordan algebra $J$ over a set of free generators $X$ (see [2]). Consider the subalgebra $R_{B}(J)$ of $\operatorname{End}_{K}(B)$ generated by all right multiplications $R_{B}(a), a \in J$. It is known that the algebra $R_{B}(J)$ does not depend on the choice of $X$. We call $M(J)=R_{B}(J)$ the universal multiplicative enveloping algebra of $J$.

If the algebra $J$ is generated by elements $x_{1}, \ldots, x_{m}$ then the algebra $M(J)$ is known to be generated by the operators $R\left(x_{i}\right), R\left(x_{i} x_{j}\right), 1 \leq i, j \leq m$ (see [2]).

We will use the following well known relations satisfied by the right multiplications of a Jordan algebra (see [2]):

$$
\begin{aligned}
& R(y) R(z) R(x)+R(x) R(z) R(y)+R((x . y) \cdot z) \\
& \quad=R(y) R(x . z)+R(x) R(y \cdot z)+R(z) R(x \cdot y) \\
& \quad=R(x . y) R(z)+R(y . z) R(x)+R(z \cdot x) R(y) .
\end{aligned}
$$


Skosirskii proved the following theorem (see Theorem 1.1 in [8]):

Theorem 2.1 (Skosirskii). Let $J$ be a Jordan algebra generated by elements $x_{1}, \ldots$, $x_{m}$ and let $N=2 m+1$. Then $M(J)$ is spanned by operators $R\left(a_{1}\right) \ldots R\left(a_{k}\right)$, where $a_{i} \in J$ and $k \leq N$.

To prove the second theorem we will need to strengthen this result.If we combine the above mentioned result by Skosirskii and Proposition 1.1 in [8], we obtain the following

Proposition 2.1. Let $J$ be a Jordan algebra generated by elements $x_{1}, \ldots, x_{m}$ and let $N=2 m+1$. Then there exists a natural number $f(m)$ such that $M(J)$ is spanned by operators $U(a, b) R\left(a_{1}\right) \ldots R\left(a_{k}\right)$, where $a, b, a_{i}$ are Jordan words in $x_{1}, \ldots, x_{m}$, $k \leq N$, and $\operatorname{deg}\left(a_{1}\right)+\ldots+\operatorname{deg}\left(a_{k}\right) \leq f(m)$.

Remark 2.1. From the proposition it follows that for arbitrary words $b_{1}, \ldots, b_{r}$ in the generators $x_{1}, \ldots, x_{m}$, the operator $\pi=R\left(b_{1}\right) \ldots R\left(b_{r}\right)$ is a linear combination of operators $R\left(a_{1}\right) \ldots R\left(a_{k}\right)$ where $k \leq N, a_{i}$ are Jordan words in $x_{1}, \ldots, x_{m}$, at most two of the words $a_{1}, \ldots, a_{k}$ have length $\geq f(m)$ and each $R\left(a_{1}\right) \ldots R\left(a_{k}\right)$ has the same composition as $\pi$.

Theorem 2.2. $G K \operatorname{dim}(M(J)) \leq 2 G K \operatorname{dim}(J)$.

Proof. Let us suppose that $J$ is generated by elements $x_{1}, \ldots, x_{m}$. Then the algebra $M(J)$ is generated by operators $R\left(x_{i}\right), R\left(x_{i} . x_{j}\right)$. Let $V$ be the linear span of $\left\{x_{1}, \ldots, x_{m}\right\}$ and let $W$ be the linear span of $\left\{R\left(x_{i}\right), R\left(x_{i} \cdot x_{j}\right)\right\}$. So $\operatorname{dim} W=M \leq m(m+1))$.

Let us denote as before $V^{n}$ and $W^{n}$ the vector spaces spanned by words $x_{i_{1}} \ldots x_{i_{r}}$, in $V$ and operators $R\left(a_{1}\right) \ldots R\left(a_{r}\right)$ in $W$, where $r \leq n, a_{i}=x_{j_{i}}$ or $a_{i}=x_{j_{i}} . x_{h_{i}}$. There are only finitely many words of length $\leq f(m)$. To be precise, there are no more than $H(m)=m+m^{2}+m^{3}+\ldots+m^{f(m)-1}$ of them.

For each pair $a, b$ of words, there are finitely many operators in the form

$U(a, b) R\left(a_{1}\right) \ldots R\left(a_{t}\right)$, with $t \leq N$ and $a_{1}, \ldots, a_{t}$ words of length $\leq f(n)$.

So the above proposition assures that for every $n \geq N, \operatorname{dim}\left(W^{n}\right) \leq C \cdot\left(\operatorname{dim} V^{n}\right)^{2}$, where $C$ is some constant (it depends only on $m$ and $N$, but not on $n$ ). Consequently $G K \operatorname{dim}(M(J)) \leq 2 G K \operatorname{dim}(J)$.

Remark 2.2. The above result can be also proved for alternative algebras. Its proof follows the same lines and ideas used in Theorem 2.2 but, instead of the modified version of the Skosirskii result, a similar result for alternative algebras due to I. Shestakov is used.

Lemma 2.1 (I. Shestakov,[6]). Let $A$ be an alternative algebra generated by the ordered set of elements $X=\left\{x_{i} \mid i \in I\right\}$. Then the universal multiplication algebra of $A, M(A)$, can be generated by elements of one of the following types:

$$
L(a) R(a), R\left(x_{i_{1}}\right) R\left(x_{i_{2}}\right) \ldots R\left(x_{i_{k}}\right) R(a), L\left(x_{j_{1}}\right) R\left(x_{j_{2}}\right) R\left(x_{j_{3}}\right) \ldots R\left(x_{j_{m}}\right) R(b)
$$

where $a, b \in A, \quad x_{h} \in X, \quad i_{1}<i_{2}<\ldots<i_{k}, \quad j_{1}<j_{2}<\ldots<j_{m}, \quad k \geq 1$, and $m \geq 2$.

Theorem 2.3. Let $A$ be a finitely generated alternative algebra and let $M(A)$ denote its multiplication algebra. Then $G K \operatorname{dim}(M(A)) \leq 2 G K \operatorname{dim}(A)$. 
Example 2.1. Let $H(O, 3)$ be the exceptional 27-dimensional algebra over $K$ and let $J=H(O, 3) \otimes K[t]$. Then $G K \operatorname{dim}(J)=1$ (because $H(O, 3)$ is finite-dimensional and $G K \operatorname{dim}(F[t])=1)$. Let $B$ be a free Jordan bimodule over $J$ and let $A$ be the subalgebra of $M(J)=R_{B}(J)$ generated by all multiplications $R_{B}(a)$, where $a$ is an element of $H(O, 3) \otimes 1$.

Jacobson proved (see [2]) that $M(H(O, 3)) \cong M_{27}(K)$. Since $A$ is an homomorphic image of the universal multiplicative algebra $M(H(O, 3))$, we have that $A \cong M_{27}(K)$.

We say that an element of the free Jordan algebra $f$ is a central polynomial for a Jordan algebra $J$ if an arbitrary value of $f$ on $J$ lies in the associative center of $J$. Y. Medvedev (see [5]) constructed a central multilinear polynomial $f$ for the free Jordan algebra which is not identically zero in $H(O, 3)$. It is easy to see that $f(J)=1 \otimes K[t]$.

Hence $1 \otimes K[t]=f(J) \subseteq f(J+B)$, so an arbitrary element $c \in 1 \otimes K[t]$ lies in the associative center of $J+B$. Hence $R_{B}($ a.c $)=R_{B}(a) R_{B}(c)=R_{B}(c) R_{B}(a)$ for an arbitrary element $a \in J$. This implies that $R_{B}(J)=A\left[R_{1 \otimes t}\right]$. Therefore $G K \operatorname{dim}\left(R_{B} J\right)=1$.

This example shows that the inequality in Theorem 3.2 may be strict, that is, it is possible that $G K \operatorname{dim}(M(J))<2 G K \operatorname{dim}(J)$.

Example 2.2. If $O$ denotes the octonions algebra and $A=O \otimes K[t]$, then we can prove in the same way that we did in the previous example, that $\operatorname{GK\operatorname {dim}}(M(A))=$ $\operatorname{GKdim}(A)=1$.

Example 2.3. If $J=K[X]$, then $M(J) \cong K[X, Y]$ (see [2]). Consequently $G K \operatorname{dim}(J)=1$ and $G K \operatorname{dim} M(J)=2$. That is, in this case $G K \operatorname{dim}(M(J))=$ $2 \operatorname{GKdim}(J)$.

This second example suggests that in the case of special Jordan algebras, the inequality given in Theorem 3.2 is in fact an equality.

Theorem 2.4. Let $J$ be a finitely generated special Jordan algebra and let $A$ be its associative enveloping algebra. If $M(J)$ is the universal multiplicative enveloping algebra, then $G K \operatorname{dim}(M(J))=2 G K \operatorname{dim}(J)=2 G K \operatorname{dim}(A)$.

Proof. We can consider $J$ with unit element and a finite set of generators $\left\{1, x_{1}, \ldots\right.$, $\left.x_{m}\right\}$ of $J$ and $A$. Let $V$ be the linear span of them, let $V^{n}$ be the associative product of $V n$-times and let $V^{\cdot n}$ be the Jordan product of $V n$-times.

Let $M=A \otimes_{K} A$. Then $M$ is a $J$-bimodule via $(x \otimes y) a=\frac{1}{2}(a x \otimes y+x \otimes a y)$.

Let $R_{M}(J)=\left\langle R_{M}(a) \mid a \in J\right\rangle$. It would be enough to prove that $G K \operatorname{dim}\left(R_{M}(J)\right)$ $\geq 2 G K \operatorname{dim}(J)$.

Since $V^{\bullet n} \subseteq V^{n}$, we can take a basis of $V^{n}$ extending a basis of $V^{\bullet n}$.

If $\left\{b_{i} \mid i \in I\right\}$ is a basis of $V^{\bullet n}$, then these elements are independent as elements of $A$. Let us show that the system $\left\{R\left(b_{i}\right) R\left(b_{j}\right)\right\}$ with $(i, j) \in I \times I$ and $i \leq j$ is a linearly independent set of $R_{M}(J)$.

In fact, an equality $\sum_{i \leq j} \lambda_{i, j} R\left(b_{i}\right) R\left(b_{j}\right)=0$ implies (when we apply it to $1 \otimes 1$ ) that $\sum_{i \leq j} \lambda_{i, j}\left(b_{i} \otimes b_{j}+b_{j} \otimes b_{i}+1 \otimes b_{i} b_{j}+b_{i} b_{j} \otimes 1\right)=0$, which yields $\lambda_{i, j}=0$ for any $(i, j) \in I \times I$ with $i \leq j$, since $\left\{b_{i} \otimes b_{j} \mid(i, j) \in I \times I, i \leq j\right\}$ is a linearly independent system in $A \otimes A$. 
Let $\tilde{V}$ be the linear span of $\left\{R(1), R\left(x_{1}\right), \ldots, R\left(x_{m}\right), R\left(x_{i} . x_{j}\right), i \leq j\right\}$ The algebra $R_{M}(J)$ is generated by $\tilde{V}$ and we have proved that

$$
\operatorname{dim} \tilde{V}^{n} \geq \frac{1}{2}\left(\operatorname{dim} V^{\bullet n}\right)\left(\operatorname{dim} V^{\bullet n}+1\right) .
$$

So $G K \operatorname{dim}\left(R_{M}(J)\right) \geq 2 G K \operatorname{dim}(J)$.

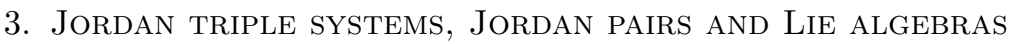

If $J$ is a Jordan algebra, $J$ can be considered as a triple system via:

$\{x y z\}=(x . y) \cdot z+(y . z) \cdot x-(x . z) \cdot y$ where . denotes the Jordan product in $J$.

We will denote by $J^{T}$ the Jordan algebra $J$ when it is considered as a triple system.

We have also a Jordan pair $P=\left(J^{-}, J^{+}\right)$associated with $J$, where $J^{-}=J^{+}=J$ and $\left\{x^{-}, y^{+}, z^{-}\right\}$and $\left\{x^{+}, y^{-}, z^{+}\right\}$are the triple products defined before.

It is clear that if $J$ is finitely generated as a triple system or as a Jordan pair, it is also finitely generated as a Jordan algebra (it can be generated by the same set of elements and products in triple systems and Jordan pairs can be expressed with the Jordan product).

Lemma 3.1. Let $J$ be a Jordan algebra finitely generated by $v_{1}, \ldots, v_{m}$. Then $J^{T}$ is generated as a triple system by all products of $v_{1}, \ldots, v_{m}$ of length $\leq 2$.

Proof. We know that if $J$ is generated by $v_{1}, \ldots, v_{m}$, then its multiplication algebra $R(J)$ is generated by $R\left(v_{i}\right)$ and $R\left(v_{i} \cdot v_{j}\right)$.

Let us consider an element of $J$ that is a product of length $\geq 4$. Then this element can be represented in the form: $a=\sum v_{i} R() R.(.) \ldots R($.$) , where at least$ two multiplication operators appear in each summand of the expression. So $a$ can be rewritten in the form: $a=\sum a_{j} R\left(a_{j}^{\prime}\right) R\left(a_{j}^{\prime \prime}\right)$ with $a_{j}^{\prime}, a_{j}^{\prime \prime} \in\left\{R\left(v_{i}\right), R\left(v_{h} v_{l}\right)\right\}$.

$\operatorname{But}\left(a_{j} \cdot a_{j}^{\prime}\right) \cdot a_{j}^{\prime \prime}=\frac{1}{2}\left\{a_{j}^{\prime} a_{j} a_{j}^{\prime \prime}\right\}+\frac{1}{2}\left\{a_{j} a_{j}^{\prime} a_{j}^{\prime \prime}\right\}$.

Thus all products of length $\geq 3$ can be expressed by using triple products.

Theorem 3.1. (1) Let $J$ be a Jordan algebra and let $J^{T},(J, J)$ be the triple system and the Jordan pair respectively associated to $J$. Then $J$ is finitely generated if and only if $J^{T}$ is finitely generated if and only if $(J, J)$ is finitely generated.

(2) $G K \operatorname{dim}(J)=G K \operatorname{dim}\left(J^{T}\right)=G K \operatorname{dim}((J, J))$.

Proof. The proof of (1) follows immediately from the previous lemma. So let us prove (2).

Since it is clear that $G K \operatorname{dim}\left(J^{T}\right)=G K \operatorname{dim}((J, J))$, we only need to prove that $G K \operatorname{dim}(J)=G K \operatorname{dim}\left(J^{T}\right)$.

Let $V$ be the linear span of a generator system $\left\{v_{1}, \ldots, v_{m}\right\}$ of $J$. Then, according to the previous lemma, the linear span $W$ of $\left\{v_{i}, v_{j} . v_{h}\right\}$ generates $J^{T}$.

If $n$ is a natural number, $V^{n}$ denotes the linear span of all products of length $\leq n$ in $v_{1}, \ldots, v_{m}$ and if $n$ is an odd natural number, then $W^{n}$ denotes the linear span of all product in $J^{T}$ of length (odd) $\leq n$ in the elements $v_{i}, v_{j} \cdot v_{h}$.

Let us note that only products of odd length in $J^{T}$ are defined.

Now it is enough to notice that $\operatorname{dim}_{K} W^{n} \leq \operatorname{dim}_{K} V^{2 n} \leq \operatorname{dim}_{K} W^{2 n-1}$ for every odd natural number $n, n \geq 3$. 
Consequently:

$$
\begin{aligned}
G K \operatorname{dim}\left(J^{T}\right) & =\limsup _{n \rightarrow \infty, \text { nodd }} \frac{\ln \left[\operatorname{dim} W^{n}\right]}{\ln n} \\
& \leq \limsup _{n \rightarrow \infty} \frac{\ln \left[\operatorname{dim} V^{2 n}\right]}{\ln n}=\limsup _{n \rightarrow \infty} \frac{\ln \left[\operatorname{dim} V^{2 n}\right]}{\ln [2 n]} \frac{\ln [2 n]}{\ln n}=G K \operatorname{dim}(J) .
\end{aligned}
$$

That is, $G K \operatorname{dim}\left(J^{T}\right) \leq G K \operatorname{dim}(J)$.

Conversely,

$$
\begin{aligned}
& G K \operatorname{dim}(J)=\limsup _{n \rightarrow \infty} \frac{\ln \left[\operatorname{dim} V^{n}\right]}{\ln n} \leq \limsup _{n \rightarrow \infty} \frac{\ln \left[\operatorname{dim} W^{2 n-1}\right]}{\ln [2 n-1]} \frac{\ln [2 n-1]}{\ln n} \\
& =G K \operatorname{dim}\left(J^{T}\right) \text {. }
\end{aligned}
$$

Now we will study the relation between Jordan pairs and Lie algebras.

If $L=L_{-1} \oplus L_{0} \otimes L_{1}$ is a short graded Lie algebra (that is $\left[L_{i}, L_{j}\right] \subseteq L_{i+j}$ and $L_{i}=0$ if $\left.|i|>1\right)$, then $\left(L_{-1}, L_{1}\right)$ is a Jordan pair with the law:

$$
\left\{x_{\epsilon}, y_{-\epsilon}, z_{\epsilon}\right\}=\left[\left[x_{\epsilon}, y_{-\epsilon}\right], z_{\epsilon}\right]
$$

where $\epsilon= \pm 1$.

The space $L_{0}$ acts as derivations of the Jordan pair $P=\left(L_{-1}, L_{1}\right)$, where $\operatorname{Der}(P)=\left\{D=\left(D_{+}, D_{-}\right) \mid D_{\epsilon}\left\{x_{\epsilon} y_{-\epsilon} z_{\epsilon}\right\}=\left\{D_{\epsilon}\left(x_{\epsilon}\right) y_{-\epsilon} z_{\epsilon}\right\}+\left\{x_{\epsilon} D_{-\epsilon}\left(y_{-\epsilon}\right) z_{\epsilon}\right\}\right.$ $+\left\{x_{\epsilon} y_{-\epsilon} D_{\epsilon}\left(z_{\epsilon}\right)\right\}$.

The original Lie algebra can be recovered from the Jordan pair by the KantorKoecher-Tits construction : If $P=\left(V_{+}, V_{-}\right)$is a Jordan pair and $L_{0}$ is a Lie subalgebra of $\operatorname{Der}(P)$, we get a Lie algebra $L=L_{-1} \oplus L_{0} \oplus L_{1}=V_{-} \oplus L_{0} \oplus V_{+}$ with the product given by:

$$
\left[x_{\epsilon}, y_{\epsilon}\right]=0, \quad\left[D, x_{\epsilon}\right]=D_{\epsilon}\left(x_{\epsilon}\right), \quad\left[D, D^{\prime}\right]_{\epsilon}=\left[D_{\epsilon}, D_{\epsilon}^{\prime}\right]
$$

and

$$
\left[x_{+}, x_{-}\right]=D_{x_{+}, x_{-}}
$$

where $\left(D_{x_{+}, x_{-}}\right)_{+}\left(z_{+}\right)=\left\{x_{+} x_{-} z_{+}\right\}$and $\left(D_{x_{+}, x_{-}}\right)_{-}\left(z_{-}\right)=-\left\{x_{-} x_{+} z_{-}\right\}$.

Theorem 3.2. Let $L=L_{-1} \oplus L_{0} \oplus L_{1}$ be a short graded Lie algebra finitely generated with $L_{0}=\left[L_{-1}, L_{1}\right]$ and let $P=\left(L_{-1}, L_{1}\right)$ be the associated Jordan pair. Then $G K \operatorname{dim}(P)=G K \operatorname{dim}\left(\left(L_{-1}, L_{1}\right)\right)$.

Proof. Let $X=X^{-} \cup X^{+}$be a finite set of generators of $P$ and let $V$ be its linear span. For each natural number $n$ we will denote by $V^{[n]}$ the linear span of products in $L$ of length $\mathrm{n}$ and for each odd natural number $V^{(n)}$ will denote the linear span of all products in $P$ of length $n$.

Clearly $V^{[n]}=V^{(n)}$ for every odd natural number, so

$$
\begin{aligned}
\operatorname{GKdim}(P) & =\limsup _{n \rightarrow \infty, \text { nodd }} \frac{\ln \left[\operatorname{dim} V+\operatorname{dim} V^{(3)}+\ldots+\operatorname{dim} V^{(n)}\right]}{\ln n} \\
& \leq \limsup _{n \rightarrow \infty} \frac{\ln \left[\operatorname{dim} V+\operatorname{dim} V^{[2]}+\ldots+\operatorname{dim} V^{[n]}\right]}{\ln n}=\operatorname{GKdim}(L) .
\end{aligned}
$$

If $n$ is an odd natural number, then $V^{[n]}=\left[V^{[n-1]}, V\right]$, so

$$
\operatorname{dim} V^{[n]} \leq M \cdot \operatorname{dim} V^{[n-1]}=M \cdot \operatorname{dim} V^{(n-1)},
$$

where $M=\operatorname{dim} V$. 
Consequently

$$
\begin{aligned}
G K \operatorname{dim}(L) & =\limsup _{n \rightarrow \infty} \frac{\ln \left[\operatorname{dim} V+\operatorname{dim} V^{[2]}+\ldots+\operatorname{dim} V^{[n]}\right]}{\ln n} \\
& \leq \limsup _{n \rightarrow \infty, n o d d} \frac{\ln \left[(1+M)\left(\operatorname{dim} V+\operatorname{dim} V^{(3)}+\ldots+\operatorname{dim} V^{(n)}\right]\right.}{\ln n} \\
& =G K \operatorname{dim}(P) .
\end{aligned}
$$

\section{ACKNOWLEDGEMENT}

The author is deeply grateful to Professors I. Shestakov and E. Zelmanov for their kind and useful comments and suggestions.

\section{REFERENCES}

1. W. Borho and H. Kraft, Uber die Gelfand-Kirillov dimension, Math. Ann., 220 (1976), 1-24. MR 54:367

2. N. Jacobson, Structure and Representation of Jordan Algebras, A.M.S. Colloq. Publ., 39, Providence (1968). MR 40:4330

3. G. Krause and T.H. Lenagan, Growth of Algebras and Gelfand-Kirillov dimension, Research Notes in Math., vol. 116, Pitman, (1985). MR 86g:16001

4. K. McCrimmon, The Russian revolution in Jordan Algebras, Algebras, Groups and Geometries, 1, (1984), 1-61. MR 86j:17028

5. Y. Medvedev, Free Jordan algebras, Algebra i Logica, 27 n.2, (1988), 172-200. MR 90c:17046

6. I.P. Shestakov, Absolute zero divisors and radicals of finitely generated alternative Algebras, Algebra i Logika, 15, n.5, (1976), 585-602. MR 58:22218

7. V.G. Skosirskii, On nilpotence in Jordan and right alternative Algebras, Algebra i Logika, 18, n.1, (1979), 73-85.

8. V.G. Skosirskii, Radicals in Jordan algebras, Sibirsk. Matem. Zhurnal, 29, no.2, (1988), 154166.

9. K.A. Zhevlakov, A.M. Slin'ko, I.P. Shestakov, A.I. Shirshov, Rings that are nearly associative, Academic Press, New York (1982). MR 83i:17001

Department of Mathematics, University of Oviedo, 33.007 Oviedo, Spain

E-mail address: chelo@@inon.ccu.uniovi.es 